\title{
STUDY OF HEAT TRANSFER WITH NONLINEAR THERMAL RADIATION ON SINUSOIDAL MOTION OF MAGNETIC SOLID PARTICLES IN A DUSTY FLUID
}

\author{
M. M. BHATTi \\ Shanghai Institute of Applied Mathematics and Mechanics, \\ Shanghai University, Shanghai China, \\ e-mails: muhammad09@shu.edu.cn, mubashirme@yahoo.com \\ A. Zeeshan, R. Ellahi \\ Department of Mathematics and Statistics, \\ International Islamic University, Islamabad Pakistan
}

[Received 30 May 2016. Accepted 20 june 2016]

\begin{abstract}
In this article, heat transfer with nonlinear thermal radiation on sinusoidal motion of magnetic solid particles in a dust Jeffrey fluid has been studied. The effects of Magnetohydrodynamic (MHD) and hall current are also taken under consideration. The governing equation of motion and energy equation are modelled with help of Ohms law for fluid and dust phases. The solutions of the resulting ordinary coupled partial differential equations are solved analytically. The impact of all the physical parameters of interest such as Hartmann number, slip parameter, Hall parameter, radiation parameter, Prandtl number, Eckert number and particle volume fraction are demonstrated mathematically and graphically. Trapping mechanism is also discussed in detail by drawing contour lines. The present analysis affirms many interesting behaviours, which permit further study on solid particles motion with heat and mass transfer.

KEY WORDS: Heat transfer, Thermal radiation, magnetic solid particle, dusty fluid.
\end{abstract}

\section{Nomenclature}

\begin{tabular}{|c|l|}
\hline$\tilde{U}, \tilde{V}$ & velocity components $(\mathrm{m} / \mathrm{s})$ \\
\hline$\tilde{X}, \tilde{Y}$ & Cartesian coordinate $(\mathrm{m})$ \\
\hline$C$ & Volume fraction density \\
\hline
\end{tabular}

\footnotetext{
${ }^{*}$ Corresponding author e-mails: muhammad09@shu.edu.cn, mubashirme@yahoo.com
} 


\begin{tabular}{|c|l|}
\hline$\tilde{P}$ & pressure in fixed frame $\left(\mathrm{N} / \mathrm{m}^{2}\right)$ \\
\hline$\tilde{a}$ & wave amplitude $(\mathrm{m})$ \\
\hline$M$ & Hartman number \\
\hline$m$ & Hall parameter \\
\hline$\tilde{b}$ & width of the channel $(\mathrm{m})$ \\
\hline$\tilde{c}$ & wave velocity $(\mathrm{m} / \mathrm{s})$ \\
\hline $\mathrm{Ec}$ & Eckert number \\
\hline $\operatorname{Pr}$ & Prandtl number \\
\hline $\operatorname{Re}$ & Reynolds number \\
\hline$\tilde{t}$ & Time $(\mathrm{s})$ \\
\hline$c$ & effective heat capacity $(\mathrm{J} / \mathrm{K})$ \\
\hline $\mathbf{B}$ & magnetic field $(\mathrm{T})$ \\
\hline$\tilde{k}$ & mean absorption coefficient \\
\hline$S$ & Drag force \\
\hline $\mathrm{k}$ & Thermal conductivity \\
\hline$N_{r}$ & Radiation parameter \\
\hline $\mathrm{e}$ & Electron charge \\
\hline $\mathrm{n}$ & Number of electron density \\
\hline$T$ & temperature $(\mathrm{K})$ \\
\hline $\mathbf{E}$ & Electric field $(\mathrm{V} / \mathrm{m})$ \\
\hline$\tilde{\mathbf{V}}$ & Velocity field $(\mathrm{m} / \mathrm{s})$ \\
\hline$Q$ & volume flow rate $\left(\mathrm{m}^{4} / \mathrm{s}\right)$ \\
\hline $\mathbf{S}$ & Stress tensor \\
\hline $\mathbf{J}$ & current density $(\mathrm{A})$ \\
\hline
\end{tabular}

\section{Greek symbols}

\begin{tabular}{|c|l|}
\hline$\theta$ & Dimensionless temperature \\
\hline$\lambda$ & Wavelength $(\mathrm{m})$ \\
\hline$\mu_{s}$ & Viscosity of the fluid $\left(\mathrm{Ns} / \mathrm{m}^{2}\right)$ \\
\hline$\varphi$ & Amplitude ratio \\
\hline$\sigma$ & Electrical conductivity $(\mathrm{S} / \mathrm{m})$ \\
\hline $\bar{\sigma}$ & Stefan-Boltzmann constant \\
\hline$\varpi_{T}$ & Thermal equilibrium time \\
\hline
\end{tabular}




\begin{tabular}{|c|l|}
\hline$\varpi_{v}$ & Relaxation time of the particle \\
\hline$\beta$ & slip parameter \\
\hline$\rho$ & Fluid density $\left(\mathrm{kg} / \mathrm{m}^{3}\right)$ \\
\hline$\lambda_{1}$ & Relaxation time \\
\hline$\lambda_{2}$ & Retardation time \\
\hline$\gamma$ & Shear rate \\
\hline$\phi$ & Stream function \\
\hline
\end{tabular}

\section{Subscripts}

\begin{tabular}{|c|l|}
\hline$f$ & Fluid phase \\
\hline$p$ & Particulate phase \\
\hline
\end{tabular}

\section{Introduction}

Heat transfer with thermal radiation is an important topic of research due to its significant applications in biomedical engineering and different kinds of medical treatment, such as thermal therapeutic process. Infrared radiation technique is one of the most useful procedures, that is very much helpful for making heat treatment in different parts of human body. This type of procedure is preferable in heat therapy, because with the help of infrared radiation it is easily attainable to heat the human body on affected parts. Heat therapy is found to be very helpful and useful in various treatments, such as widespread chronic pain (or fibromyalgia), permanent shortening of muscle (contracture), muscle spasms and bursitis (inflammation of a bursa). Several experimental and theoretical investigations have been carried out by different scientists to analyse the influence of nonlinear thermal radiation with different fluid models [1-4]. For instance, Hayat and Qasim [5] investigated the influence of Joule heating and thermal radiation on MHD flow of non-Newtonian Maxwell fluid in the presence of thermophoresis. Rashidi et al. [6] studied the heat transfer with thermal radiation of micropolar fluid through a porous media and found the solution with the help of differential transform method. Sheikholeslami et al. [7] examined the effect of heat transfer and thermal radiation on MHD nanofluid flow of two phase model. Akbar et al. [8] analysed the radiation effects on stagnation point flow of MHD nanofluid towards a stretching sheet under the influence of heat transfer and convective boundary condition. Some more studies on the said topic can be found from the list of refs. [9-18] and several therein. 
In a human body, due to symmetrical contraction and expansion of smooth muscles, produce a series wave like motion known as peristaltic motion (or sinusoidal motion). This process is found in the oesophagus when a bolus of food is swallowed, movement of Chyme in small intestine, transport of cilia, human lymphatic system and movement of sperm etc. Different kinds of devices that works on the principle of peristaltic phenomena, such as finger pumps, heart lung machine and roller pumps. At present, this topic has been investigated by various researchers with different assumptions and fluid models. Some of the pertinent studies can be found from the refs. [19-21]. Mekheimer [22] studied the peristaltic motion of couple stress fluids under the influence of endoscopic effect. Mekheimer and Elmaboud [23] examined the peristaltic flow with endoscopic effect through a porous medium. Abbas et al. [24] investigated three dimensional peristaltic motion of non-Newtonian hyperbolic tangent fluid having flexible walls. Recently, Ellahi et al. [25] studied the peristaltic flow of couple stress fluid in a non-uniform rectangular duct having compliant walls.

On the other hand, the theoretical and mathematical study of solid particle motion is very much favourable to understand various kinds of physical problems, such as movement of solid particles in a liquid, movement of slurries in nuclear and chemical process, fluidization, powder technology and lunar ash flows etc. The sedimentation of solid particles in a Newtonian or non-Newtonian fluid is of great interest, due to its applications in medicine, oceanography and chemical engineering process. Hung and Brown [26] introduced the solid particle motion in a two dimensional channel with the help of peristaltic phenomena. Later, Mekheimer et al. [27] described the peristaltic phenomena of particle-fluid suspension in a two dimensional planar channel. Mekheimer and Abd Elmaboud [28] described the peristaltic motion of particle fluid suspension in a uniform and non-uniform annulus. Some more studies on the said topic can be found from the list of refs. [29-31] and several therein.

The study of electrically conducting fluids in the presence of thermal radiation and magnetic field are found in various applications such as solar power technology, electric power generation, magneto-plasma dynamics, electric generators, linear motors and nuclear engineering [32-34]. In various microfluidics problems, MHD is examined as fluid pump to create a non-pulsating flow and continuous flow in different geometrical designs. Magnetohydrodynamics is also very much helpful to control the flow due to the influence of Lorentz force. Ellahi et al. [35] investigated the effects of Magnetohydrodynamics on peristaltic flow of Jeffrey fluid through a porous rectangular duct. Hayat et al. [36] explored the effects of wall properties and magnetic field on peristaltic flow of Maxwell fluid with heat and mass transfer. Mekheimer [37] studied the 
influence of magnetic field on peristaltic motion of couple stress fluid in a nonuniform and uniform channel. Some more studies on Magnetohydrodynamics can be viewed in the references [38-46]. According to the best of author's knowledge, no such attempt has been made on heat transfer with nonlinear thermal radiation on magnetic solid particle motion of dusty fluid under the influence of Hall Effect.

Motivated from the above analysis, the aim of the present study is to analyse the heat transfer with nonlinear thermal radiation on sinusoidal motion of magnetic solid particle in dusty fluid under the influence of Hall. The governing flow problem is modelled, using long wavelength and zeros Reynolds number approximation. The solutions of the resulting differential equations for fluid and dust phases are solved analytically and exact solutions have been presented for velocity and temperature profiles. The expression for pressure rise is calculated numerically with the help of computer generated codes.

\section{Mathematical formulation}

Consider the sinusoidal wave motion of magnetic solid particle in dusty Jeffrey fluid in a two dimensional planar channel, under the influence of Hall current. The fluid is electrically conducting by an external magnetic field. Cartesian coordinate system is chosen in such a way i.e., $\tilde{X}$-axis is considered along the wave propagation, while $\tilde{Y}$-axis is taken normal to it, as shown in Fig. 1:

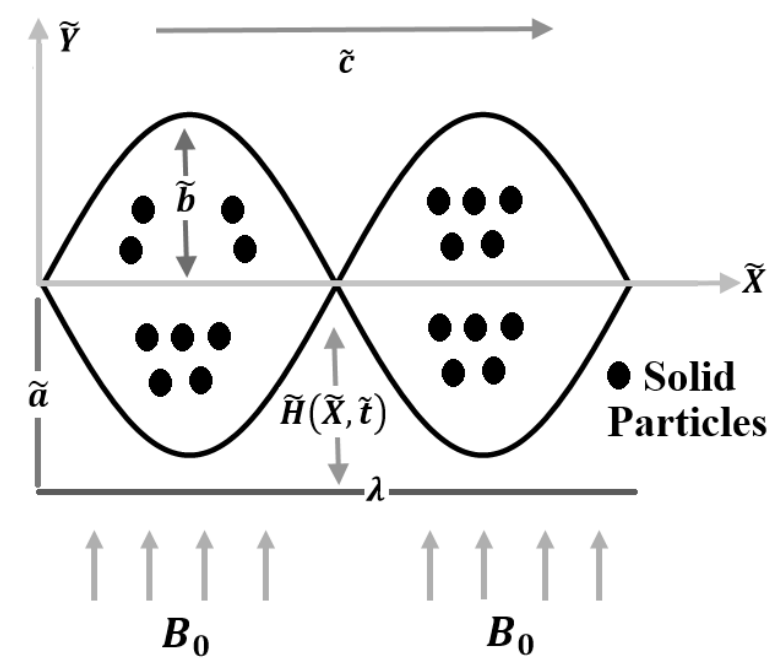

Fig. 1. Geometry of the problem 
The geometry of the wall can be written as:

$$
\tilde{H}(\tilde{X}, \tilde{t})=\tilde{a}+\tilde{b} \sin \frac{2 \pi}{\lambda}(\tilde{X}-\tilde{c} \tilde{t}) .
$$

The magnetic field is considered of the following, form [35]:

$$
\mathbf{B}=\left(0, B_{0}\right),
$$

with the help of Ohm's Law, we have:

$$
\mathbf{J}=\sigma\left[\mathbf{E}+\tilde{\mathbf{V}} \times \mathbf{B}-\frac{1}{\mathrm{e}_{\mathrm{n}}}(\mathbf{J} \times \mathbf{B})\right]
$$

The governing equation of continuity, linear momentum and energy equations for fluid phase and dust phase, can be written as [27]:

For fluid phase:

$$
\frac{\partial \tilde{U}_{f}}{\partial \tilde{X}}+\frac{\partial \tilde{V}_{f}}{\partial \tilde{Y}}=0
$$

(5) $\quad(1-C) \rho_{f}\left(\frac{\partial \tilde{U}_{f}}{\partial \tilde{t}}+\tilde{U}_{f} \frac{\partial \tilde{U}_{f}}{\partial \tilde{X}}+\tilde{V}_{f} \frac{\partial \tilde{U}_{f}}{\partial \tilde{Y}}\right)$

$$
\begin{aligned}
=-(1-C) \frac{\partial \tilde{P}}{\partial \tilde{X}}+(1-C)\left(\frac{\partial}{\partial \tilde{X}} \mathrm{~S}_{\tilde{X} \tilde{X}}+\frac{\partial}{\partial \tilde{Y}} \mathrm{~S}_{\tilde{X} \tilde{Y}}\right) \\
+\frac{C S}{\varpi_{v}}\left(\tilde{U}_{p}-\tilde{U}_{f}\right)+\mathbf{J} \times \mathbf{B},
\end{aligned}
$$

(6) $\quad(1-C) \rho_{f}\left(\frac{\partial \tilde{V}_{f}}{\partial \tilde{t}}+\tilde{U}_{f} \frac{\partial \tilde{V}_{f}}{\partial \tilde{X}}+\tilde{V}_{f} \frac{\partial \tilde{V}_{f}}{\partial \tilde{Y}}\right)$

$$
\begin{aligned}
=-(1-C) \frac{\partial \tilde{P}}{\partial \tilde{Y}}+(1-C)\left(\frac{\partial}{\partial \tilde{X}} \mathrm{~S}_{\tilde{Y} \tilde{X}}+\frac{\partial}{\partial \tilde{Y}} \mathrm{~S}_{\tilde{Y} \tilde{Y}}\right) & \\
& +\frac{C S}{\varpi_{v}}\left(\tilde{V}_{p}-\tilde{V}_{f}\right),
\end{aligned}
$$

(7) $(1-C) \rho_{f} c\left(\frac{\partial T_{f}}{\partial \tilde{t}}+\tilde{U}_{f} \frac{\partial T_{f}}{\partial \tilde{X}}+\tilde{V}_{f} \frac{\partial T_{f}}{\partial \tilde{Y}}\right)$

$$
\begin{aligned}
=\mathrm{k}(1-C) \frac{\partial^{2} T_{f}}{\partial \tilde{Y}^{2}}+\frac{\rho_{p} c_{p} C}{\varpi_{T}}\left(T_{p}-T_{f}\right) & +\frac{C S}{\varpi_{v}}\left(\tilde{U}_{f}-\tilde{U}_{p}\right)^{2} \\
& +\mathrm{S}_{\tilde{X} \tilde{Y}}(1-C) \frac{\partial \tilde{U}_{f}}{\partial \tilde{Y}}-\frac{\partial q_{r}}{\partial \tilde{Y}},
\end{aligned}
$$


for dust phase:

$$
\begin{gathered}
\frac{\partial \tilde{U}_{p}}{\partial \tilde{X}}+\frac{\partial \tilde{V}_{p}}{\partial \tilde{Y}}=0 \\
C \rho_{p}\left(\frac{\partial \tilde{U}_{p}}{\partial \tilde{t}}+\tilde{U}_{p} \frac{\partial \tilde{U}_{p}}{\partial \tilde{X}}+\tilde{V}_{p} \frac{\partial \tilde{U}_{p}}{\partial \tilde{Y}}\right)=-C \frac{\partial \tilde{P}}{\partial \tilde{X}}+\frac{C S}{\varpi_{v}}\left(\tilde{U}_{f}-\tilde{U}_{p}\right), \\
C \rho_{p}\left(\frac{\partial \tilde{V}_{p}}{\partial \tilde{t}}+\tilde{U}_{p} \frac{\partial \tilde{V}_{p}}{\partial \tilde{X}}+\tilde{V}_{p} \frac{\partial \tilde{V}_{p}}{\partial \tilde{Y}}\right)=-C \frac{\partial \tilde{P}}{\partial \tilde{Y}}+\frac{C S}{\varpi_{v}}\left(\tilde{V}_{f}-\tilde{V}_{p}\right) \\
\rho_{p} C c\left(\frac{\partial T_{p}}{\partial \tilde{t}}+\tilde{U}_{p} \frac{\partial T_{p}}{\partial \tilde{X}}+\tilde{V}_{p} \frac{\partial T_{p}}{\partial \tilde{Y}}\right)=\frac{\rho_{p} C c}{\varpi_{T}}\left(T_{f}-T_{p}\right)
\end{gathered}
$$

The mathematical expression for the drag coefficient and the empirical relation for the viscosity of the suspension can be described as:

$$
\begin{aligned}
& S=\frac{9 \mu_{0}}{2 a^{2}} \tilde{\lambda}(C), \quad \tilde{\lambda}(C)=\frac{4+3 \sqrt{8 C-3 C^{2}}+3 C}{(2-3 C)^{2}}, \\
& \mu_{s}=\frac{\mu_{0}}{1-\chi C}, \quad \chi=0.07 e^{\left[2.49 C+\frac{1107}{T} e^{-1.69 C}\right]} .
\end{aligned}
$$

The nonlinear radiative heat flux can be written as:

$$
q_{r}=-\frac{4 \bar{\sigma}}{3 \bar{k}} \frac{\partial T^{4}}{\partial \tilde{Y}}=-\frac{16 \bar{\sigma} T^{3}}{3 \bar{k}} \frac{\partial T}{\partial \tilde{Y}} .
$$

The stress tensor of Jeffrey fluid, which is defined as:

$$
\mathbf{S}=\frac{\mu_{s}}{1+\lambda_{1}}\left(\dot{\gamma}+\lambda_{2} \ddot{\gamma}\right)
$$

Now it is convenient to describe the transformation variable from fixed frame to wave frame

$$
\tilde{x}=\tilde{X}-\tilde{c} \tilde{t}, \tilde{y}=\tilde{Y}, \tilde{u}_{f, p}=\tilde{U}_{f, p}-\tilde{c}, \tilde{v}_{f, p}=\tilde{V}_{f, p}, \tilde{p}=\tilde{P} .
$$


Introducing the following non-dimensional quantities:

$$
\begin{aligned}
& \tilde{x}=\frac{x}{\lambda}, \quad \tilde{y}=\frac{y}{\tilde{a}}, \quad \tilde{u}_{f, p}=\frac{u_{f, p}}{\tilde{c}}, \quad \tilde{v}_{f, p}=\frac{v_{f, p}}{\tilde{c} \delta}, \quad h=\frac{\tilde{H}}{\tilde{a}}, \quad \phi=\frac{\tilde{b}}{\tilde{a}}, \\
& p=\frac{\tilde{a}^{2}}{\lambda \tilde{c} \mu_{s}} \tilde{p}, \quad \operatorname{Re}=\frac{\rho \tilde{a} \tilde{c}}{\mu_{s}}, \quad \theta_{f, p}=\frac{T_{f, p}-T_{0}}{T_{1}-T_{0}}, \quad \operatorname{Pr}=\frac{\mu_{s} c}{\mathrm{k}}, \\
& \mathrm{Ec}=\frac{\tilde{c}^{2}}{c\left(T_{1}-T_{0}\right)}, \quad N_{1}=\frac{S \tilde{a}^{2}}{\varpi_{v} \mu_{s}}, \quad N_{r}=\frac{16 \bar{\sigma} T^{3}}{3 \bar{k} \mu_{s} c(1-C)}, \\
& m=\frac{\sigma B_{0}}{\mathrm{e}_{\mathrm{n}}}, \quad \beta=\frac{\bar{\beta}}{b_{0}} .
\end{aligned}
$$

using Eqs (15) and (16) in Eq. (4) to Eq. (11), and taking the approximation of long wavelength and low creeping flow regime. The resulting equations for fluid phase can be written as:

$$
\begin{aligned}
& \frac{d p}{d x}=\frac{1}{1+\lambda_{1}} \frac{\partial^{2} u_{f}}{\partial y^{2}}-\frac{M^{2}}{1+m^{2}}\left(u_{f}+1\right)+\frac{N C}{(1-C)}\left(u_{p}-u_{f}\right) \\
& \left(\frac{1}{\operatorname{Pr}}+N_{r}\right) \frac{\partial^{2} \theta_{f}}{\partial y^{2}}=-\frac{\mathrm{Ec}}{1+\lambda_{1}}\left(\frac{\partial u_{f}}{\partial y}\right)^{2}-\frac{\mathrm{Ec}}{N(1-C)}\left(\frac{d p}{d x}\right)^{2}
\end{aligned}
$$

For particulate phase, it can be written as:

$$
u_{p}=u_{f}-\frac{d p}{d x} \frac{1}{N_{1}}
$$

$$
\theta_{p}=\theta_{f}
$$

and their corresponding non-dimensional slip boundary conditions, are:

$$
\begin{aligned}
& u_{f}^{\prime}=\theta_{f}=0 \text { at } y=0 \text { and } \\
& u_{f}=-1-\frac{\beta}{1+\lambda_{1}} u_{f}^{\prime}, \theta_{f}=1 \text { at } y=h=1+\phi \sin 2 \pi x .
\end{aligned}
$$


Study of Heat Transfer with Nonlinear Thermal Radiation ...

\section{Problem solution}

The exact solution of Eq. (17) to Eq. (20) can be written as:

$u_{f}=\frac{\sqrt{1+\lambda_{1}}\left((1-c) N^{2}+\frac{d p}{d x}\right) \cosh \left[h \sqrt{1+\lambda_{1}} N\right]}{(-1+C) N^{2}\left(\sqrt{1+\lambda_{1}} \operatorname{Cosh}\left[h \sqrt{1+\lambda_{1}} N\right]+N \beta \sinh \left[h \sqrt{1+\lambda_{1}} N\right]\right)}$

(22) $-\frac{\frac{d p}{d x} \sqrt{1+\lambda_{1}} \cosh \left[\sqrt{1+\lambda_{1}} N y\right]}{(-1+C) N^{2}\left(\sqrt{1+\lambda_{1}} \operatorname{Cosh}\left[h \sqrt{1+\lambda_{1}} N\right]+N \beta \sinh \left[h \sqrt{1+\lambda_{1}} N\right]\right)}$

$+\frac{\sqrt{1+\lambda_{1}} N\left(N-C N^{2}+\frac{d p}{d x}\right) \beta \sinh \left[h \sqrt{1+\lambda_{1}} N\right]}{(-1+C) N^{2}\left(\sqrt{1+\lambda_{1}} \operatorname{Cosh}\left[h \sqrt{1+\lambda_{1}} N\right]+N \beta \sinh \left[h \sqrt{1+\lambda_{1}} N\right]\right)}$,

$u_{p}=\frac{\sqrt{1+\lambda_{1}}\left((1-c) N^{2}+\frac{d p}{d x}\right) \cosh \left[h \sqrt{1+\lambda_{1}} N\right]}{(-1+C) N^{2}\left(\sqrt{1+\lambda_{1}} \operatorname{Cosh}\left[h \sqrt{1+\lambda_{1}} N\right]+N \beta \sinh \left[h \sqrt{1+\lambda_{1}} N\right]\right)}$

$-\frac{\frac{d p}{d x} \sqrt{1+\lambda_{1}} \cosh \left[\sqrt{1+\lambda_{1}} N y\right]}{(-1+C) N^{2}\left(\sqrt{1+\lambda_{1}} \operatorname{Cosh}\left[h \sqrt{1+\lambda_{1}} N\right]+N \beta \sinh \left[h \sqrt{1+\lambda_{1}} N\right]\right)}$

(23)

$$
\begin{aligned}
& +\frac{\sqrt{1+\lambda_{1}} N\left(N-C N^{2}+\frac{d p}{d x}\right) \beta \sinh \left[h \sqrt{1+\lambda_{1}} N\right]}{(-1+C) N^{2}\left(\sqrt{1+\lambda_{1}} \operatorname{Cosh}\left[h \sqrt{1+\lambda_{1}} N\right]+N \beta \sinh \left[h \sqrt{1+\lambda_{1}} N\right]\right)} \\
& -\frac{d p}{d x} \frac{1}{N_{1}},
\end{aligned}
$$




$$
\begin{aligned}
& \theta_{f, p}=\left(\left(\frac{\mathrm{Ec}}{1+\lambda_{1}}\left(\frac{d p}{d x}\right) \operatorname{Pr}(h-y)\left(-1+2 h\left(1+\lambda_{1}\right) N^{2} y\right)\right.\right. \\
& +2(-1+c) N^{3} y\left(2(-1+c) N\left(1+N_{r} \operatorname{Pr}\right)\right. \\
& \left.+\operatorname{Ec} h\left(\frac{d p}{d x}\right)^{2} \operatorname{Pr}(-h+y)\right)\left(-1+\left(1+\lambda_{1}\right) N^{2} \beta^{2}\right) \\
& -y\left(\frac{\mathrm{Ec}}{1+\lambda_{1}}\left(\frac{d p}{d x}\right) \operatorname{Pr}+4(-1+C)^{2} N^{4}\left(1+N_{r} \operatorname{Pr}\right)\right. \\
& -2(-1+C) \operatorname{Ec} N^{3}\left(\frac{d p}{d x}\right)^{2} \operatorname{Pr}(h-y)+ \\
& +4(-1+C)^{2}\left(1+\lambda_{1}\right) N^{6}\left(1+N_{r} \operatorname{Pr}\right) \beta^{2} \\
& \left.-2(-1+C) \operatorname{Ech}\left(1+\lambda_{1}\right) N^{5}\left(\frac{d p}{d x}\right)^{2} \operatorname{Pr}(h-y) \beta^{2}\right) \cosh \left[2 h \sqrt{1+\lambda_{1}} N\right] \\
& +\frac{\mathrm{Ec}}{1+\lambda_{1}} h\left(\frac{d p}{d x}\right)^{2} \operatorname{Pr} \cosh \left[2 \sqrt{1+\lambda_{1}} N y\right] \\
& -4(-1+C) \sqrt{1+\lambda_{1}} N^{4} y\left(2(-1+C) N\left(1+N_{r} \operatorname{Pr}\right)\right. \\
& \left.\left.+\operatorname{Ech}\left(\frac{d p}{d x}\right)^{2} \operatorname{Pr}(-h+y)\right) \beta \sinh \left[2 h \sqrt{1+\lambda_{1}} N\right]\right) / \\
& \left(4 ( - 1 + C ) ^ { 2 } h N ^ { 4 } ( 1 + N _ { r } \operatorname { P r } ) \left(1-\left(1-\lambda_{1}\right) N^{2} \beta^{2}\right.\right. \\
& +1+\left(1+\lambda_{1}\right) N^{2} \beta^{2} \cosh \left[2 h \sqrt{1+\lambda_{1}} N\right] \\
& \left.\left.\left.+2 \sqrt{1+\lambda_{1}} N \beta \sinh \left[2 h \sqrt{1+\lambda_{1}} N\right]\right)\right)\right)
\end{aligned}
$$


The volume flow rate is given by:

$$
Q=(1-C) \int_{0}^{h} u_{f} \mathrm{dy}+C \int_{0}^{h} u_{p} \mathrm{dy} .
$$

The pressure gradient $d p / d x$

is obtained after solving the above Eq. (25), we get:

$$
\begin{aligned}
\frac{d p}{d x}=-\{(-1 & +C) \sqrt{1+\lambda_{1}} N^{3} N_{1}(h+Q)\left(\sqrt{1+\lambda_{1}} \cosh \left[h \sqrt{1+\lambda_{1}} N\right]\right. \\
& \left.\left.+N \beta \sinh \left[h \sqrt{1+\lambda_{1}} N\right]\right)\right\}
\end{aligned}
$$

$$
\begin{aligned}
& \times\left\{h \sqrt{1+\lambda_{1}} N\left((-1+C) C N^{2}-N_{1}\right) \cosh \left[h \sqrt{1+\lambda_{1}} N\right]\right. \\
& \left.+\left(N_{1}-h\left(1+\lambda_{1}\right) N^{2}\left(-(-1+C) C N^{2}+N_{1}\right) \beta\right) \sinh \left[h \sqrt{1+\lambda_{1}} N\right]\right\}^{-1} .
\end{aligned}
$$

The non-dimensional pressure rise $\Delta p$ is evaluated numerically with the help of computer generated codes, by using the following expression:

$$
\Delta p=\int_{0}^{1} \frac{d p}{d x} \mathrm{dx},
$$

where $N=\sqrt{\frac{M^{2}}{1+m^{2}}}$.

The expression for dimensionless stream function satisfying equation of continuity is defined as:

$$
\tilde{U}_{f, p}=\frac{\partial \varphi_{f, p}}{\partial y}, \quad \tilde{V}_{f, p}=-\frac{\partial \varphi_{f, p}}{\partial x} .
$$

\section{Numerical results and discussion}

In this section, the behaviour of all the pertinent parameters of interest are discussed for velocity profile, temperature profile, pressure rise and trapping phenomena. Velocity profile and temperature profile are sketched for Newtonian and non-Newtonian as special case of our study. In order to brought out the inclusion of different emerging parameters, computational software Mathematica has been used to visualize the behaviour of all the parameters through 
graphs. One can clearly observe, that the magnitude of the velocity profile, temperature profile and pressure rise is smaller, when the fluid is non-Newtonian $\left(\lambda_{1} \neq 0\right)$, as compared to Newtonian fluid $\left(\lambda_{1}=0\right)$.

\subsection{Velocity profile}

Figures 2 and 3 represent the behaviour of velocity profile against Hartmann number $(M)$, Hall parameter $(m)$, particle volume fraction $(C)$ and slip parameter $\beta$. It depicts from Fig. (2a), that when the magnetic field is applied, then it opposes the flow due to the influence of Lorentz force and hence the velocity of the fluid diminishes. From Fig. (2b) it can be observed, that when the hall parameter increases, then magnitude of the velocity profile increases. It can be notice from Fig. (3a), that when the ratio of particle volume fraction $(C)$ increases, then it leads to decrease the velocity of the fluid. Figure (3b) is drawn against various values of slip parameter. It can be seen from this figure, that velocity of the profile shows opposite behaviour near the walls, while its behaviour become versatile in the centre of the channel.
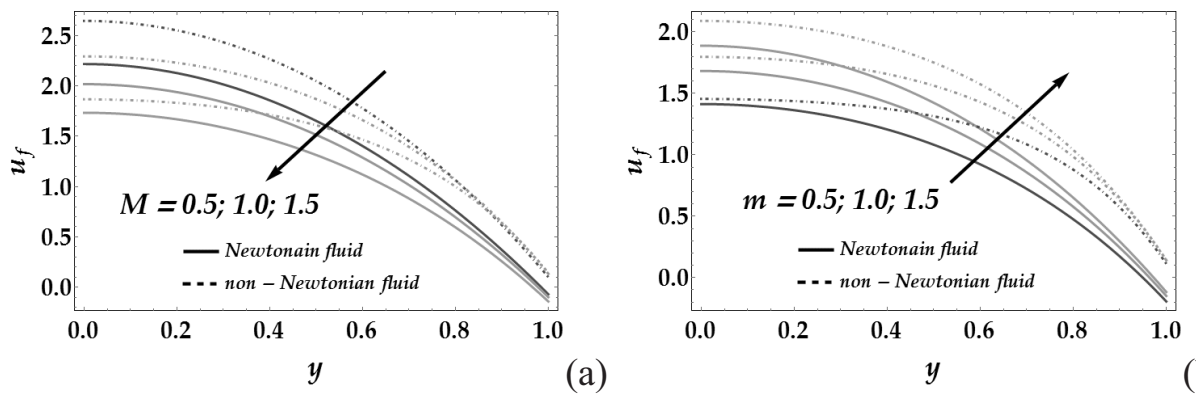

Fig. 2. Velocity profile for various values of $M$ and $m$ when $\phi=0.7, \beta=0.1$, $C=0.3, Q=2, m=0.5$
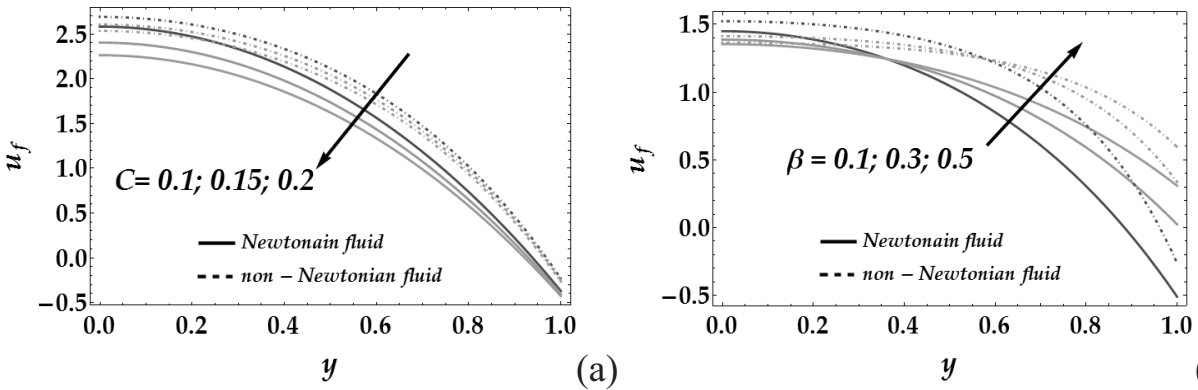

Fig. 3. Velocity profile for various values of $C$ and $\beta$ when $\phi=0.7, M=1, \beta=0.1$, $Q=2, m=0.5$ 

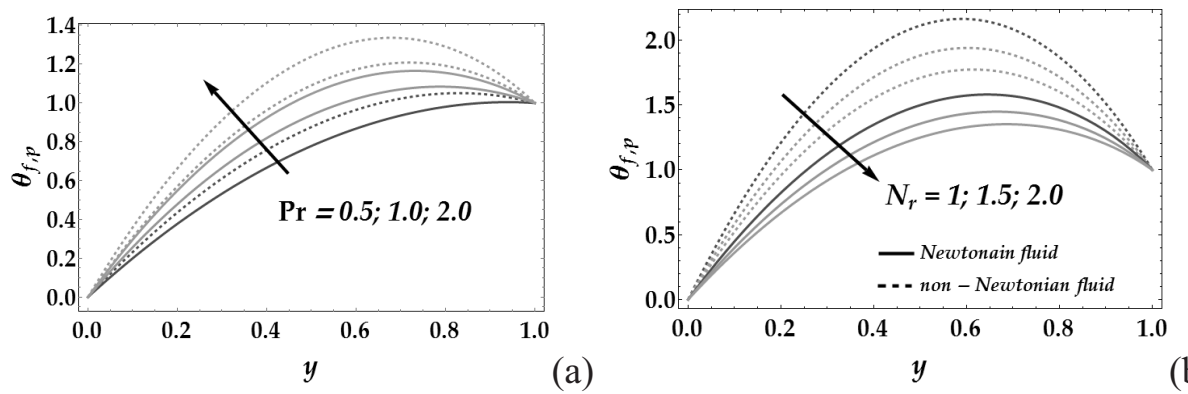

(b)

Fig. 4. Temperature profile for various values of $\operatorname{Pr}$ and $N_{r}$ when $\phi=0.7, M=1$, $\beta=0.1, C=0.3, Q=2, m=0.5, N_{r}=1, \mathrm{Ec}=0.5$
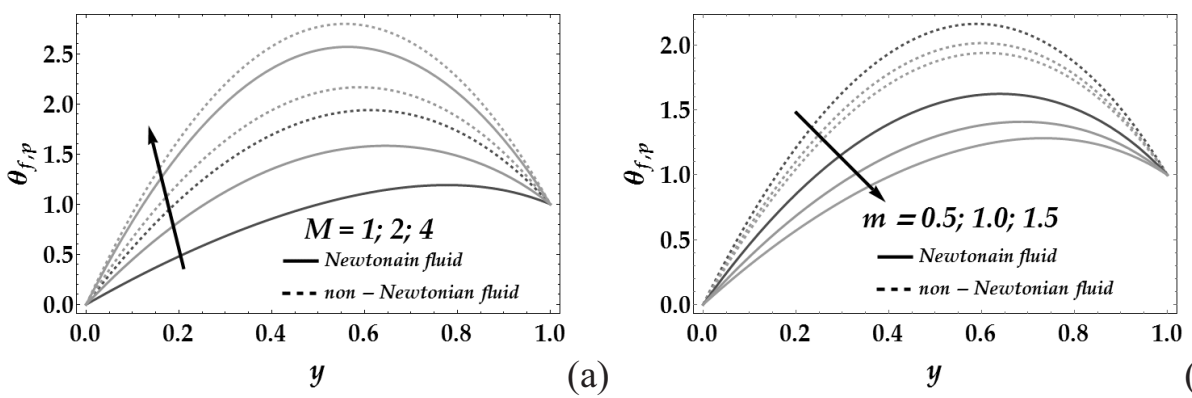

Fig. 5. Temperature profile for various values of $M$ and $m$ when $\phi=0.7, \beta=0.1$, $C=0.3, Q=2, m=0.5, N_{r}=1, \mathrm{Ec}=0.5, \operatorname{Pr}=0.5$

\subsection{Temperature profile}

Figure 4 a shows the impact of Prandtl number on temperature profile. It can be observed from this figure, that temperature profile enhances due to the influence of Prandtl number. From Fig. $4 \mathrm{~b}$ it can be scrutinized, that the impact of thermal radiation $(C)$ tends to reduce the temperature profile. It can be examined from Fig. 5 a, that when the Hartmann number $(M)$ increases, then the magnitude of the temperature profile increases, while its behaviour has been observed opposite for Hall parameter, as shown in Fig. 5 b. Figure 6 a is plotted against Eckert number (Ec). From this figure, we can observe, that when the Eckert number increases, then it tends to increase the temperature profile. Figure $6 \mathrm{~b}$ describes the behaviour of slip effect on temperature profile. This figure shows that temperature profile behaves as decreasing function, due to influence of slip effects, whereas the behaviour of temperature profile remains similar, when the particle volume fraction $C$ increases (see Fig. 7). 

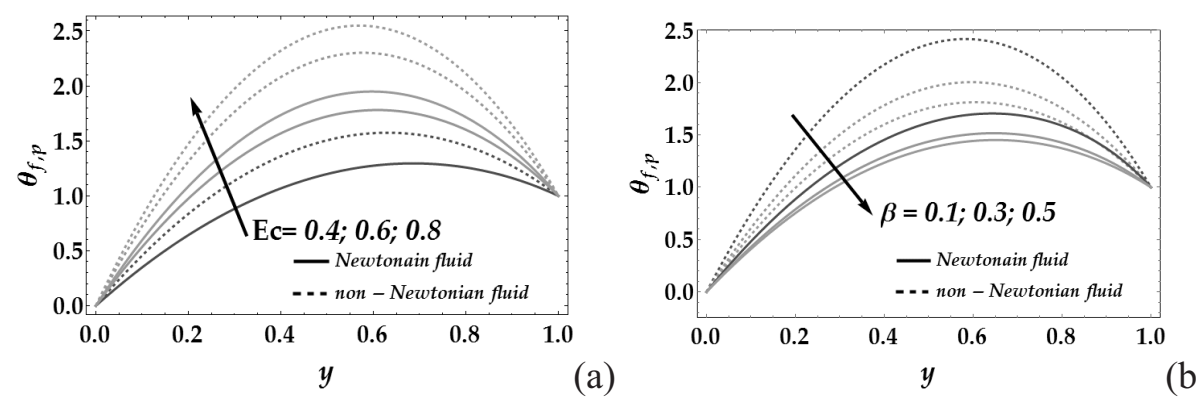

Fig. 6. Temperature profile for various values of Ec and $\beta$ when $\phi=0.7, M=1$, $\beta=0.1, C=0.3, Q=2, m=0.5, N_{r}=1, \operatorname{Pr}=0.5$

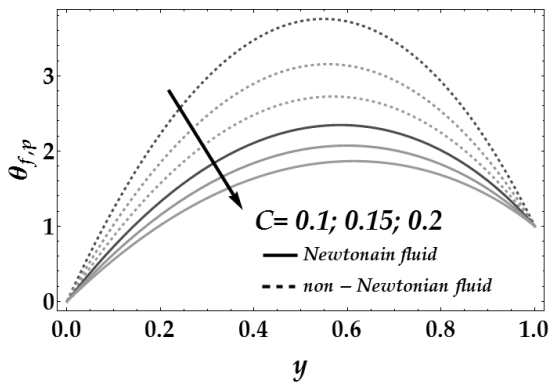

Fig. 7. Temperature profile for various values of $C$ when $\phi=0.7, M=1, \beta=0.1$, $Q=2, m=0.5, N_{r}=1, \mathrm{Ec}=0.5, \operatorname{Pr}=0.5$

\subsection{Pumping characteristics}

To discuss the pumping characteristics, Figs 8 and 9 are plotted against volume flow rate $(Q)$. Peristaltic pumping is very helpful in transporting a variety of biological fluids in a human body. Various devices have been introduced in biomedical engineering, that works on the mechanism of peristalsis. It is observed from Fig. 8 a, that the impact of magnetic field tends to decrease the pumping rate in co-pumping and free pumping regions, whereas its attitude is observed opposite in retrograde pumping region. It depicts from Fig. $8 \mathrm{~b}$ and Fig. 9 a, that when the particle volume fraction $(C)$ and Hall parameter $(m)$ increases, then the pumping rate decreases in retrograde pumping region and while the pumping rate shows similar behaviour in co-pumping and free pumping regions. It can be examine from Fig. $9 \mathrm{~b}$, that the nature of pumping rate is versatile in free pumping region, whereas opposite attitude has been noticed in retrograde pumping and co-pumping regions. 

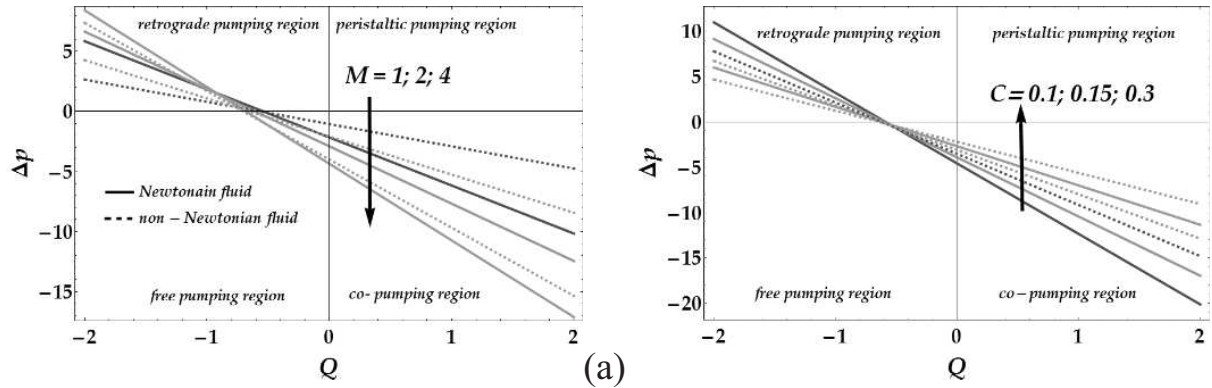

(b)

Fig. 8. Pressure rise vs volume flow rate for various values of $M$ and $C$ when $\phi=0.7, \beta=0.1, C=0.3, Q=2, m=0.5$
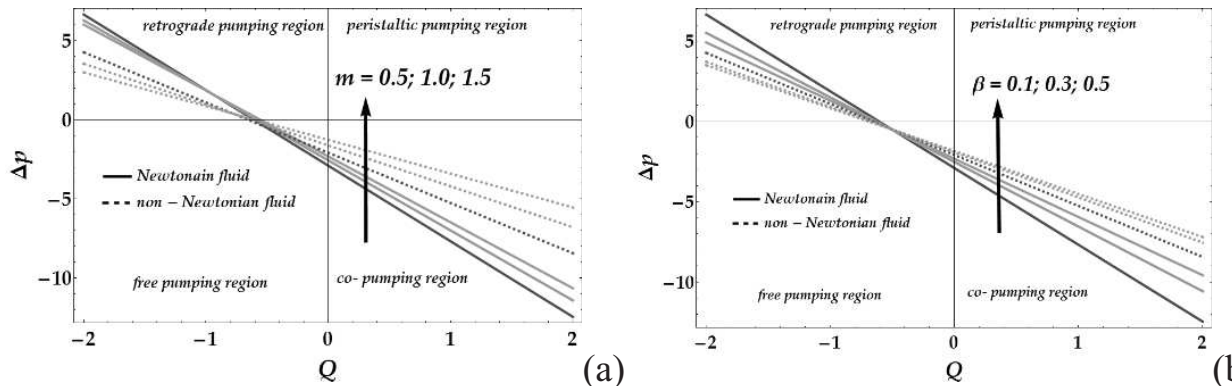

Fig. 9. Pressure rise vs volume flow rate for various values of $m$ and $\beta$ when $\phi=0.7$,

$$
M=1, \beta=0.1, C=0.3, Q=2
$$

\subsection{Trapping mechanism}

Another most engrossing part of this section is trapping, which is taken under consideration by drawing contour lines. Basically, it is the formulation of internally circulating bolus, enclosed by contour lines. This type of mechanism is very helpful in variety of blood flow problems. Examples include formation of thrombus and the movement of food bolus in gastrointestinal tract. It can be examined from Fig. 10, that when the slip parameter $(\beta)$ increases, then the magnitude of trapped bolus reduces, while the number of bolus also reduces. It is clear from Fig. 11, that due to the influence of Hall parameter $(m)$, the number of tapped bolus increases. It reveals from Fig. 12, that when the particle volume fraction increases, then it does not create any major influence on trapped bolus. It can be notice from Fig. 13, that when that Hartmann number $(M)$ increases, then size of trapped bolus decreases very rapidly, while the bolus also disappears. It is infect due to the Lorentz force that is originated, when the magnetic field is applied, which tends to oppose the flow and as a 

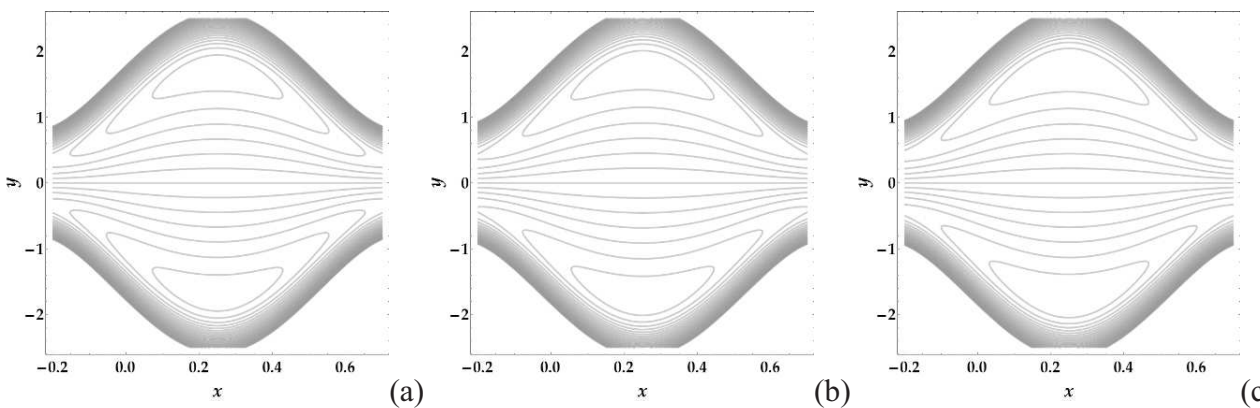

Fig. 10. Contour lines for various values of $\beta$,(a) 0.1 , (b) 0.3 , (c) 0.5 , when $\phi=0.7$, $M=1, C=0.3, Q=2, m=0.5$
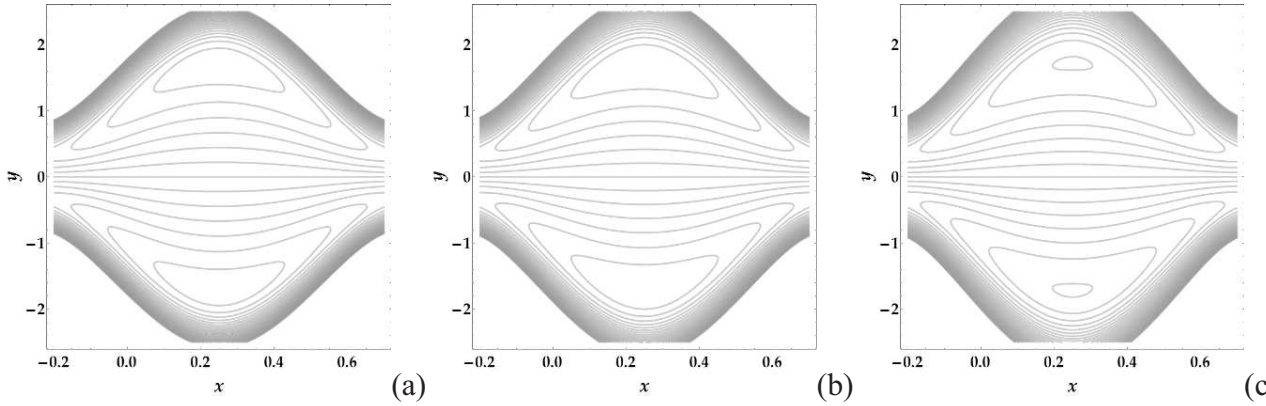

Fig. 11. Contour lines for various values of $m$ (a) 0.5 , (b) 1 , (c) 1.5, when $\phi=0.7$, $M=1, \beta=0.1, C=0.3, Q=2$
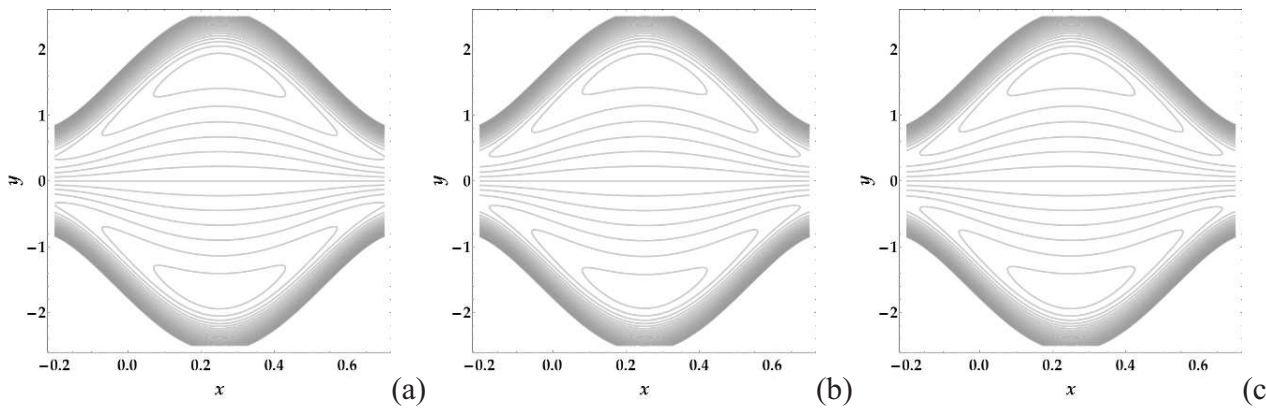

Fig. 12. Contour lines for various values of $C$ (a) 0.1 , (b) 0.15 , (c) 0.2 , when $\phi=0.7$, $M=1, \beta=0.1, Q=2, m=0.5$

result, the magnitude of trapping bolus reduces and also the number of bolus decreases. 

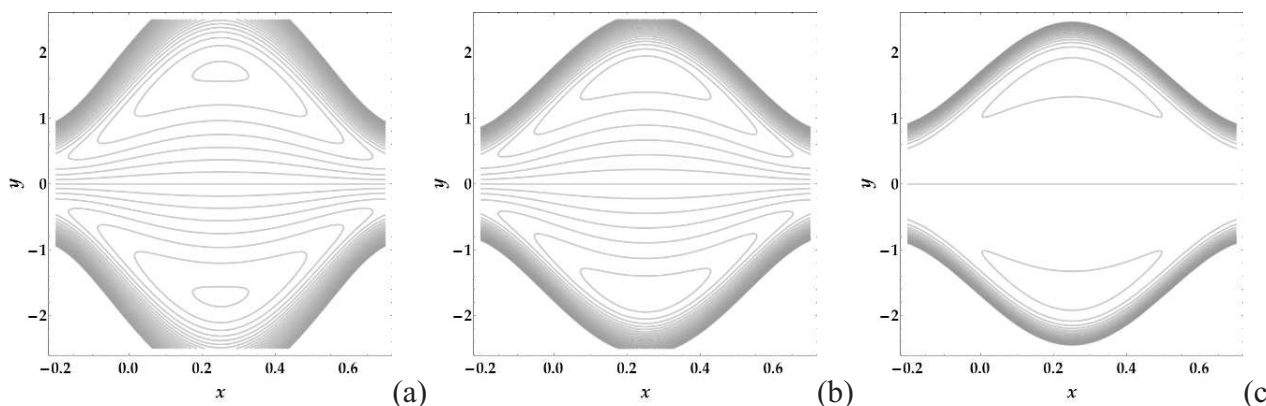

Fig. 13. Contour lines for various values of $M$ (a) 1 , (b) 2 , (c) 3.8 , when $\phi=0.7$, $\beta=0.1, C=0.3, Q=2, m=0.5$

\section{Conclusion}

In this paper, heat transfer with nonlinear thermal radiation on sinusoidal motion of magnetic solid particle in dust fluid, under the Hall effect has been investigated. The governing flow problem is formulated under the assumption of long wavelength and zero Reynolds number approximation. The resulting coupled partial differential equations have been solved analytically and a closed form solution is obtained. The impacts of all the parameters are demonstrated mathematically and graphically for Newtonian and nonNewtonian fluid. The major outcomes of our present analysis are summarized below:

- Velocity profile diminishes, due to the influence of magnetic field and particle volume fraction, while its behaviour is opposite for hall parameter.

- Due to slip effects, velocity profile shows opposite behaviour on the walls.

- Temperature profile increases with the increment in Hartman number and Prandtl number, while it decreases due to Hall and radiation effect.

- Pressure rise show similar behaviour for particle volume fraction and slip parameter, whereas its attitude is reverse for Hartman number.

- The present analysis can also be reduced to Newtonian fluid by taking $\lambda_{1}$, as special case of our study.

\section{REFERENCES}

[1] Hayat, T., M. Awais, A. Alsaedi, A. Safdar. On Computations for Thermal Radiation in MHD Channel Flow with Heat and Mass Transfer. PloS one., 9 (2014), e86695. 1-5. 
[2] Rashidi, M. M., B. Rostami, N. Freidoonimehr, S. Abbasbandy. Free Convective Heat and Mass Transfer for MHD Fluid Flow over a Permeable Vertical Stretching Sheet in the Presence of the Radiation and Buoyancy Effects. Ain Shams Eng. J., 5 (2014), 901-912.

[3] Sheikholeslami, M., D. D. Gangi, M. M. Rashidi. Ferrofluid Flow and Heat Transfer in a Semi Annulus Enclosure in the Presence of Magnetic Source Considering Thermal Radiation. J. Taiwan Inst. Chem. Eng., 47 (2015), 6-17.

[4] Rashidi, M. M., N. V. Ganesh, A. A. Hakeem, B. Ganga. Buoyancy Effect on MHD Flow of Nanofluid Over a Stretching Sheet in the Presence of Thermal Radiation. J. Mol. Liq., 198 (2014), 234-238.

[5] Hayat, T., M. QAsim. Influence of Thermal Radiation and Joule Heating on MHD Flow of a Maxwell Fluid in the Presence of Thermophoresis. Int. J. Heat Mass Trans., 53 (2010), 4780-4788.

[6] Rashidi, M. M., S. A. Mohimanian Pour. A Novel Analytical Solution of Heat Transfer of a Micropolar Fluid through a Porous Medium with Radiation by DTM-Padé. Heat Tran.-Asian Res., 39 (2010), 575-589.

[7] Sheikholeslami, M., D. D. Ganji, M. Y. Javed, R. Ellahi. Effect of Thermal Radiation on Magnetohydrodynamics Nanofluid Flow and Heat Transfer by Means of Two Phase Model. J. Magn. Magn. Mater., 374 (2015), 36-43.

[8] Akbar, N. S., S. Nadeem, R. U. Haq, Z. H. Khan. Radiation Effects on MHD Stagnation Point Flow of Nano Fluid Towards a Stretching Surface with Convective Boundary Condition. Chin. J. Aeronaut., 26 (2013), 1389-1397.

[9] Kandelousi, M. S. Effect of Spatially Variable Magnetic Field on Ferrofluid Flow and Heat Transfer Considering Constant Heat Flux Boundary Condition. Eur. Phy. J. Pl., 129 (2014), No. 11, 1-12.

[10] Kandelousi, M. S. KKL Correlation for Simulation of Nanofluid Flow and Heat Transfer in a Permeable Channel. Phys. Lett. A., 378 (2014), No. 45, 3331-3339.

[11] Sheikholeslami, M. Effect of Uniform Suction on Nanofluid Flow and Heat Transfer over a Cylinder. J. Braz. Soc. Mech. Sci. Eng., 37 (2015), No. 6, 16231633.

[12] Sheikholeslami, M., D. D. Ganji, M. M. Rashidi. Magnetic Field Effect on Unsteady Nanofluid Flow and Heat Transfer using Buongiorno Model. J. Magn. Magn. Mater., 416 (2016), 164-173.

[13] Sheikholeslami, M., T. Hayat, A. Alsaedi. MHD Free Convection of Al 2 O 3-Water Nanofluid Considering Thermal Radiation: A Numerical Study. Int. J. Heat Mass Trans., 96 (2016), 513-524.

[14] Sheikholeslami, M., H. R. Ashorynejad, P. Rana. Lattice Boltzmann Simulation of Nanofluid Heat Transfer Enhancement and Entropy Generation. J. Mol. Liq., 214 (2016), 86-95.

[15] Sheikholeslami, M., K. Vajravelu, M. M. Rashidi. Forced Convection Heat Transfer in a Semi Annulus under the Influence of a Variable Magnetic Field. Int. J. Heat Mass Trans., 92 (2016), 339-348. 
[16] Sheikholeslami, M., R. Ellahi. Three Dimensional Mesoscopic Simulation of Magnetic Field Effect on Natural Convection of Nanofluid. Int. J. Heat Mass Trans., 89 (2015), 799-808.

[17] Sheikholeslami, M., S. Abelman. Two-phase Simulation of Nanofluid Flow and Heat Transfer in an Annulus in the Presence of an Axial Magnetic Field. Nanotech. IEEE Transac., 14 (2015), No. 3, 561-569.

[18] Kandelousi, M. S., R. Ellahi. Simulation of Ferrofluid Flow for Magnetic Drug Targeting using the Lattice Boltzmann Method. Z. Naturforsch., 70 (2015), No. 2, 115-124.

[19] Akbar, N. S., S. Nadeem. Peristaltic Flow of a Phan?Thien?Tanner Nanofluid in a Diverging Tube. Heat Tran.-Asian Res., 41 (2012), 10-22.

[20] Mekheimer, K. S., S. Z. A. Husseny, Y. A. Elmaboud. Effects of Heat Transfer and Space Porosity on Peristaltic Flow in a Vertical Asymmetric Channel. Numer. Meth. Part. D. E., 26 (2010), 747-770.

[21] Mekneimer, K. S. Non-linear Peristaltic Transport of Magnetohydrodynamic Flow in an Inclined Planar Channel. Arab. J. Sci. Eng., 28 (2003), 183-202.

[22] Mekneimer, K. S. Peristaltic Flow of a Couple Stress Fluid in an Annulus: Application of an Endoscope. Physica A, 387 (2008), 2403-2415.

[23] Mekheimer, K. S., Y. Abd Elmaboud. Peristaltic Flow through a Porous Medium in an Annulus: Application of an Endoscope. Appl. Math. Info. Sci., 2 (2008), 103-121.

[24] Abbas, M. A., Y. Q. Bai, M. M. Bhatti, M. M. Rashidi. Three Dimensional Peristaltic Flow of Hyperbolic Tangent Fluid in Non-uniform Channel Having Flexible Walls. Alexandria Eng. J., 55 (2016) 653-662.

[25] Ellahi, R., M. M. Bhatti, C. Fetecau, K. Vafai. Peristaltic Flow of Couple Stress Fluid in a Non-Uniform Rectangular Duct Having Compliant Walls. Commun. Theor. Phys., 65 (2016), 66-72.

[26] Hung, T. K., T. D. Brown. Solid-particle Motion in Two-dimensional Peristaltic Flows. J. Fluid Mech., 73 (1976), 77-96.

[27] Merheimer, K. S., E. F. EL Shehawey, A. M. Elaw. Peristaltic Motion of a Particle-fluid Suspension in a Planar Channel. Int. J. Theor. Phys., 37 (1998), 2895-2920.

[28] Mekneimer, K. S., Y. ABD Elmaboud. Peristaltic Transport of a Particlefluid Suspension through a Uniform and Non-uniform Annulus. Appl. Bio. Biomech., 5 (2008), 47-57.

[29] Saxena, M., V. P. Srivastava. Particulate Suspension Flow induced by Sinusoidal Peristaltic Waves. Jpn J. Appl. Phys., 36 (1997), 385.

[30] Mekheimer, K. S., Y. Abd Elmaboud, A. I. Abdellateef. Particulate Suspension Flow induced by Sinusoidal Peristaltic Waves through Eccentric Cylinders: Thread Annular. Int. J. Biomath., 6 (2013), 1350026.

[31] Kamel, M. H., I. M. Eldesoky, B. M. Maher, R. M. Abumandour. Slip Effects on Peristaltic Transport of a Particle-Fluid Suspension in a Planar Channel. Appl. Bio. Biomech., 2015 (2015), 1-4. 
[32] Sheikholeslami, M., D. D. Ganji, M. M. Rashidi. Ferrofluid Flow and Heat Transfer in a Semi Annulus Enclosure in the Presence of Magnetic Source Considering Thermal Radiation. J. Taiw. Inst. Chem. Eng., 47 (2015), 6-17.

[33] Sheikholeslami, M., D. D. Ganji, M. Y. Javed, R. Ellahi. Effect of Thermal Radiation on Magnetohydrodynamics Nanofluid Flow and Heat Transfer by Means of Two Phase Model. J. Magn. Magn. Mater., 374 (2015), 36-43.

[34] Sheikholeslami, M., D. D. Ganji. Unsteady Nanofluid Flow and Heat Transfer in Presence of Magnetic Field Considering Thermal Radiation. J. Braz. Soc. Mech. Sci. Eng., 37 (2015), No. 3, 895-902.

[35] Ellahi, R., M. M. Bhatti, A. Riaz, M. Sheikholeslami. Effects of Magnetohydrodynamics on Peristaltic Flow of Jeffrey Fluid in a Rectangular Duct through a Porous Medium. J. Porous Media, 17 (2014), 143-157.

[36] Hayat, T., S. Hina. The Influence of Wall Properties on the MHD Peristaltic Flow of a Maxwell Fluid with Heat and Mass Transfer. Nonlinear Anal. Real World Appl., 11 (2010), 3155-3169.

[37] Mekheimer, K. S. Peristaltic Flow of Blood under Effect of a Magnetic Field in a Non-uniform Channels. Appl. Math. Comput., 153 (2004), 763-777.

[38] Sheikholeslami, M., M. M. Rashidi, T. Hayat, D. D. Ganji. Free Convection of Magnetic Nanofluid Considering MFD Viscosity Effect. J. Mol. Liq., 218 (2016), 393-399.

[39] Sheikholeslami, M., D. D. Ganji, M. M. Rashidi. Magnetic Field Effect on Unsteady Nanofluid Flow and Heat Transfer using Buongiorno Model. J. Magn. Magn. Mater., 416 (2016), 164-173.

[40] Zeeshan, A., A. Majeed, R. Ellahi. Effect of Magnetic Dipole on Viscous Ferro-fluid Past a Stretching Surface with Thermal Radiation. J. Mol. Liq., 215 (2016), 549-554.

[41] Sheikholeslami, M., M. M. Rashidi, D. D. Ganji. Numerical Investigation of Magnetic Nanofluid Forced Convective Heat Transfer in Existence of Variable Magnetic Field using Two Phase Model. J. Mol. Liq., 212 (2015), 117-126.

[42] Sheikholeslami, M., M. M. Rashidi, D. D. Ganji. Effect of Non-uniform Magnetic Field on Forced Convection Heat Transfer of Water Nanofluid. Comput. Method. Appl. Mech. Eng., 294 (2015), 299-312.

[43] Sheikholeslami, M., D. D. Ganji. Entropy Generation of Nanofluid in Presence of Magnetic Field using Lattice Boltzmann Method. Physica A., 417 (2015), 273286.

[44] Sheikholeslami, M., S. Abelman, D. D. Ganji. Numerical Simulation of MHD Nanofluid Flow and Heat Transfer Considering Viscous Dissipation. Int. J. Heat Mass Trans., 79 (2014), 212-222.

[45] Sheikholeslami, M., D. D. Ganji. Ferrohydrodynamic and Magnetohydrodynamic Effects on Ferrofluid Flow and Convective Heat Transfer. Energy, 75 (2014), 400-410.

[46] Sheikholeslami, M., D. D. Ganji. Magnetohydrodynamic Flow in a Permeable Channel Filled with Nano Fluid. Scientia Iranica B., 21 (2014), No. 1, 203-212. 